\title{
Michael Borgolte
}

\section{„Totale Geschichte“ des Mittelalters? \\ Das Beispiel der Stiftungen}

\author{
Antrittsvorlesung
}

2. Juni 1992

Humboldt-Universität zu Berlin

Fachbereich Philosophie und Geschichtswissenschaften

Institut für Geschichtswissenschaften 
Herausgeberin:

Die Präsidentin der Humboldt-Universität zu Berlin Prof. Dr. Marlis Dürkop

Copyright: Alle Rechte liegen beim Verfasser.

\section{Redaktion:}

Christine Gorek

Forschungsabteilung der Humboldt-Universität

Unter den Linden 6

10099 Berlin

Herstellung:

Linie DREI, Agentur für Satz und Grafik

Wühlischstraße 33

10245 Berlin

Heft 4

Redaktionsschluß:

21. 6.1993 


\section{$\mathrm{D}$}

er französische Soziologe und Ethnograph Marcel Mauss hat 1925 einen Essay über Form und Funktion des Gabenaustausches in archaischen Gesellschaften publiziert, der bis zum heutigen Tag nichts von seiner Anregungskraft eingebüßt hat ${ }^{1}$. Mauss' Abhandlung beruhte auf dem Studium der Indianerstämme und Eskimos in Nordamerika sowie rezenter Kulturen der pazifischen Inselwelt, bezog aber auch vergleichend die Rechts- und Wirtschaftsordnungen im antiken Rom, in Indien und bei den Germanen ein. Grundlegend für Mauss wurde die Einsicht, daß die Geschenke in den genannten und vielen anderen Kulturen zwar der Form nach freiwillig gegeben wurden, tatsächlich jedoch obligatorisch waren ${ }^{2}$. Der Pflicht zur Gabe war nicht bloß der Schenker unterworfen, sondern sie band ebenso den Adressaten, der die Gabe nicht zurückweisen durfte und selbst erwidern mußte. Dieses System des Gabentausches beruhte auf der Vorstellung, daß mit der Sache die Seele des Schenkers selbst gegeben wurde. Ursprünglich haben freilich nicht Individuen, sondern Kollektive Clans, Stämme oder Familien - miteinander kontrahiert. Das Spektrum der Gaben ging weit über das hinaus, was ein moderner Mensch erwarten mag. Neben Gütern und Reichtümern, beweglicher und unbeweglicher Habe oder wirtschaftlich nutzbaren Objekten nannte Mauss Höflichkeiten und Festessen, Rituale, Militärdienste, Tänze und Märkte, Frauen und Kinder. Der Gabentausch in archaischen Gesellschaften betraf also nicht nur Recht und Ökonomie, sondern auch Religion, Ästhetik und Moral. Mauss bezeichnete deshalb den Gabenaustausch als ein ,totales soziales Phänomen“3. Totale soziale Phänomene seien also gleichzeitig juristische, wirtschaftliche, religiöse, ästhetische und auch morphologische Tatsachen. Sie hätten in einigen Fällen die Gesellschaft insgesamt in Gang gehalten, in anderen zumindest eine große Zahl von Institutionen bestimmt. Derartige Ganzheiten oder gesellschaftliche Systeme legte Mauss, der Soziologe, 
nachdrücklich den Historikern ans Herz: „Nichts ist unserer Meinung nach dringender und hoffnungsvoller, als ein solches Studium der totalen gesellschaftlichen Phänomene"

Die Wirkung, die Mauss erzielen wollte, beschränkte sich aber keineswegs auf die Rezeption seiner genialen sozialhistorischen Methode. Vielmehr lag ihm wohl vor allem an seinem Stoff, der nicht nur aus der Perspektive der Wissenschaft, sondern auch aus derjenigen des Lebens gewürdigt werden muß. Mauss glaubte nämlich, der von ihm entdeckte Gabenaustausch habe sich nicht auf die archaischen Kulturen beschränkt, er präge schlechthin alle Gesellschaften, vor allem aber seine eigene. Moral und Ökonomie des Gebens, Nehmens und Wiedergebens seien, wie er schrieb, einer der Felsen, auf denen unsere Gesellschaften ruhten $^{5}$. Im Frankreich der III. Republik sei ,das Thema der Gabe, der Freiwilligkeit und des Zwangs der Gabe, der Großzügigkeit und des Interesses“ wiederaufgetaucht ,wie ein beherrschendes, doch lange vergessenes Motiv"“6. Mauss bezog sich mit dieser Bemerkung zustimmend auf die Gesetzgebung zur Sozialversicherung von 1924, die er als ,schon verwirklichten Staatssozialismus“ bezeichnete 7 . Daneben hob er die Errungenschaft der Familienbeihilfen hervor, ,die unsere französischen Industriellen freiwillig und tatkräftig zugunsten kinderreicher Arbeiter entwickelt haben“" und die ,eine spontane Antwort auf das Bedürfnis der Arbeitgeber" sei, ,die Individuen an sich zu binden“. Aus seiner Analyse zog er dezidierte moralische Schlüsse: ,Wir brauchen mehr guten Willen, Großzügigkeit bei Dienstmietverträgen, bei der Wohnungsvermietung und beim Verkauf von lebenswichtigen Gütern. Und wir müssen ein Mittel finden, um die Einkünfte aus Spekulation und Wucher einzuschränken (...). So kann und soll man zu archaischen und elementaren Prinzipien zurückkehren; man wird dann Handlungsmotive entdecken, die zahlreiche Gesellschaften und Klassen noch kennen: die Freude am öffentlichen Geben; das Gefallen an ästhetischem Luxus; das Vergnügen der Gastfreundschaft und des privaten oder öffentlichen Festes ${ }^{68}$.

Wie man sieht, hat Marcel Mauss aus seinem Studium des Gabentausches und unter Bezug auf seine eigene Zeit eine soziale Utopie entwickelt. Nicht in diesem Sinne, doch im Hinblick auf 
seine wissenschaftlichen Prinzipien sind ihm bedeutende französische Historiker gefolgt. Mit seinem „Essai sur le don“ und anderen Schriften wurde Mauss zu einem der Wegbereiter einer Geschichtsbewegung, die sich selbst als „Histoire Nouvelle“ bezeichnet hat ${ }^{9}$. Nach ihrem zentralen Publikationsorgan spricht man auch von der ,Schule der Annales“. Von Anfang an haben diese Gelehrten jede sektorielle, auf bestimmte Fachdisziplinen beschränkte Geschichtsforschung abgelehnt und in der Art von Mauss ein Konzept der historischen Synthese verfochten. Im Unterschied zur älteren französischen Historie, der man eine einseitige Fixierung auf die Staaten- und Ereignisgeschichte vorwarf, wollten Gründer und Anhänger der Annales Sozialgeschichte im denkbar umfassenden Sinne betreiben. Nicht das große historische Individuum, sondern der Mensch in seinem gesellschaftlichen Umfeld rückte ins Zentrum des neuen Geschichtsbildes. Alle Wissenschaften, die zur Erkenntnis der Menschen und menschlichen Gruppen in ihrer Lebenswelt beitragen konnten, wurden zu historischen Disziplinen. Die neue Historie verstand und versteht sich als eine Wissenschaft ohne Grenzen ${ }^{10}$, sie ist das interdisziplinäre Forschungsfeld schlechthin. Waren zunächst Soziologie und Geographie die bevorzugten Partner der französischen Historiker, so traten bald Psychologie, Linguistik, Demographie, Anthropologie, Archäologie und andere hinzu.

Um 1960 begann die zweite Generation der Annales-Schule, das Konzept der neuen Geschichtsbewegung als ,histoire totale“ zu bezeichnen ${ }^{11}$. Die Wortprägung erinnert nicht zufällig an die ,,totalen sozialen Phänomene“, da man sich mit ihr ausdrücklich auf Marcel Mauss berufen hat ${ }^{12}$. In der Tradition von Mauss und anderen Vätern der Annales steht auch der bewußt hergestellte Bezug zwischen Wissenschaft und Leben, der die ,totale“ oder ,globale Geschichte" kennzeichnet. Einer der prominentesten Vertreter der Annales brachte dies noch vor kurzem in einer autobiographischen Notiz klar zum Ausdruck: „Ich wollte Staatsbürger sein, um ein besserer Historiker sein zu können, und es war mir immer darum zu tun, ein Mensch meiner Zeit zu sein, um besser ein Mann der Vergangenheit sein zu können" "13. Am Anfang jeder Geschichtsforschung steht also ein Problem ${ }^{14}$ oder eine Frage, das bzw. die die jeweilige Gegenwart des Historikers bestimmt; da 
aber die eigene Lebenswelt eine denkbar dichte Komplexität darstellt, lassen sich auch in der oder durch die Geschichte nur Lösungen finden, die in möglichst umfassender Weise Kontakte und Interferenzen der verschiedenen Wirklichkeitsbereiche berücksichtigen. Man muß interdisziplinär arbeiten und forschen, weil sich im Leben selbst alles durchdringt. Daß Totalität als Postulat freilich niemals die vollständige Entwirrung oder Erfassung der historischen Beziehungsgeflechte meinen kann, ist selbstverständlich, weil jedwede Forschung als Fortschreiten ins Unendliche definiert ist ${ }^{15}$.

In Deutschland, genauer gesagt: in Westdeutschland, wurden die Anregungen der „Nouvelle Histoire“ nur zögernd und auch nicht vollständig rezipiert. Das lag einerseits daran, daß sich die neue Geschichtsbewegung in Frankreich selbst erst nach dem Mai 1968 wirklich durchsetzen konnte und über die Grenzen des Landes hinaus populär wurde ${ }^{16}$, andererseits aber an den Traditionen der deutschen Geschichtswissenschaft. Das Wort und das Konzept der "histoire totale“ wird noch in unserer Zeit nahezu einhellig verworfen, obwohl sich doch auch die besten Köpfe der deutschen Historie um großangelegte Synthesen bemühen. In der Ablehnung der ,totalen Geschichte" sind sich die Mittelalter- und die Neuzeithistoriker einig ${ }^{17}$. Man kann ein Handbuch der frühmittelalterlichen Geschichte Europas von 1991 nehmen $^{18}$ oder auch eine „Deutsche Gesellschaftsgeschichte“ des 18. Jahrhunderts von $1987^{19}$, und findet in beiden gleichlautend die Zurückweisung der „Totalitätsutopie“; ja, schon der Anspruch auf die Erforschung der Totalität sei illegitim. Es ist das Verdienst von Otto Gerhard Oexle gezeigt zu haben, daß diese Urteile auf einem Mißverständnis der erkenntnistheoretischen Voraussetzungen beruhten. Dieses Mißverständnis habe seine Wurzeln in den Wahrnehmungsweisen der deutschen Wissenschaftstradition, die überaus stark von Rankes Auffassung der Geschichte und der Geschichtsschreibung bestimmt sei ${ }^{20}$. Beeinflußt von Rankes Objektivitätsideal - die Historie müsse zeigen, „wie es eigentlich gewesen“ ist -, habe man die ,histoire totale" fälschlich als Abbildung einer angenommenen Totalität verstanden. Dies wäre in der Tat eine Illusion, doch lägen der französischen Schule wissenschaftsgeschichtlich ganz andere Orientierungen 
zugrunde. Es sei nämlich die Gegenwart, die die Frage nach dem „Ganzen“ der Geschichte aufwerfe; und weil die Gegenwart in ihren unaufhörlichen Wandlungen dem Historiker ständig neue Fragen zuführe, sei seine Forschung auch ständig auf ein anderes Ganzes gerichtet ${ }^{21}$. Mit dieser Interpretation hat Oexle in der Tat den Kern der französischen Erkenntnislehre freigelegt, auch wenn man zugeben muß, daß einige Äußerungen der Annalesisten das beschriebene Mißverständnis begünstigt haben ${ }^{22}$.

Oexle ist auch der einzige deutsche Mediävist, der bisher das Konzept des ,totalen sozialen Phänomens“ von Marcel Mauss erprobt hat. Sein Thema war die alle Gesellschaften stark prägende Erscheinung der Memoria, also des Gedenkens Toter durch Lebende. Im Mittelalter gehörte die Memoria vorzüglich dem Bereich der Kirche, das heißt der Liturgie, an, doch durchdrang sie nahezu alle Bereiche des Lebens. Memorialquellen konnten Inschriften, Kunstwerke, Geschichtsschreibung, Testamente, Geschäftsschriftgut usw. sein. Memoria ist, wie Oexle formuliert hat, ,eine Denkform und (eine) Form des sozialen Handelns von Menschen in Gruppen, die in umfassender Weise profane und religiöse Gegebenheiten verknüpft“; sie ist „,eine Form des Denkens und Handelns, in der politische und rechtliche, wirtschaftliche, gesellschaftliche und künstlerische Gegebenheiten und Intentionen in einer umfassenden religiösen Sinngebung zusammengebunden sind“"23. Diese Charakteristik entspricht genau den Kriterien, die Mauss vor fast siebzig Jahren für die ,totalen sozialen Phänomene" genannt hatte.

In meiner heutigen Vorlesung möchte ich zeigen, daß auch die Stiftungen des Mittelalters bzw. der Vormoderne totale soziale Tatsachen gewesen sind. Dabei wird sich erweisen, daß die Stiftungen einerseits ein Sonderfall des Gabentausches waren und andererseits von der Memoria geprägt worden sind. Nicht nur methodisch, auch sachlich werden sich meine Darlegungen also den Arbeiten von Mauss und Oexle anfügen. Freilich bleiben die Stiftungen trotzdem noch etwas anderes. Dies werde ich abschließend erörtern, wenn es darum geht, ob und inwiefern die Stiftungen auch Thema einer totalen Geschichte sind oder sein können. 
Was „Stiftung“ in Vergangenheit und Gegenwart sei, entzieht sich einer einfachen Definition ${ }^{24}$. Das Phänomen freilich läßt sich in vielen Hochkulturen verifizieren: im Abendland und in den islamischen Staaten ${ }^{25}$, in der vorchristlichen Antike Roms und Griechenlands $^{26}$, aber auch im Alten Ägypten ${ }^{27}$. Andererseits haben sich die Auffassungsweisen der Stiftung in Neuzeit und Moderne auseinanderentwickelt, so daß Franzosen, Angelsachsen und Deutsche verschiedene Zugänge zur gleichen Erscheinung gefunden haben ${ }^{28}$. In Deutschland dominiert ein juristischer Ansatz, seitdem die Rechtswissenschaftler im 19. Jahrhundert um den Begriff der Stiftung gerungen haben. Auch die historische Forschung steht im Banne dieser Tradition, die es freilich kritisch zu rezipieren gilt ${ }^{29}$. Unzweifelhaft ist die Stiftung ein Rechtsinstitut. Sie wird dadurch geschaffen, daß ein Stifter die Erträge seines Vermögens einem dauernden Zweck widmet ${ }^{30}$. Das Kapital der Stiftung selbst muß also erhalten bleiben, während die Zinsen gemäß dem Stifterwillen konsumiert werden. Von der einfachen Schenkung unterscheidet sich die Stiftung dadurch, daß die Gabe nicht durch einmaligen Akt den Besitzer wechselt, sondern ständig wiederholt wird. Weil die Stiftung auf eine unbestimmte Zukunft hin angelegt ist - eigentlich sogar auf Ewigkeit -, bedarf sie einer eigenen Verwaltung. Man spricht von den „Stiftungsorganen", die das Vermögen zu erhalten und zu mehren suchen und im Namen des Stifters regelmäßig die Empfänger der Wohltaten versorgen. Schon diese kurze Beschreibung deutet an, daß die Stiftung mit den von Mauss genannten Kriterien des totalen sozialen Phänomens erfaßt werden kann. Stiftung ist nämlich nicht nur eine Einrichtung des Rechts, sondern - wie sich zeigte - auch eine der Wirtschaft. Sie entspricht überdies dem, was Mauss mit einem morphologischen Phänomen meinte ${ }^{31}$; denn die Stiftung kann nur funktionieren, wenn sie sich auf ein hochentwickeltes Rechts- und Wirtschaftssystem im Ganzen bezieht, das ihren Bestand absichert. Weitere Aspekte des Stiftungswesens erschließen sich durch Analyse der Stiftungszwecke. Im Mittelalter dienten die Stiftungen hauptsächlich dem Ausbau des Kirchenwesens bzw. der Vermehrung des Gottesdienstes, der Aufhebung oder Entschärfung sozialer Notlagen und der Entfaltung von Kunst und Wissenschaft. Das alle Stiftungstypen über- 
wölbende Motiv war freilich eine religiöse Sinngebung, die nirgendwo fehlte.

Was zunächst die Kirchen betrifft, so kann man behaupten, daß ohne die Stiftungstätigkeit der Laien schon die Mission in Mitteleuropa kaum erfolgreich gewesen wäre ${ }^{32}$. Es waren nämlich vermögende Grundherren, die auf ihrem Boden Kirchen errichteten, von denen aus die Landbevölkerung christianisiert wurde. Die Bischöfe waren auf derartige Partner angewiesen aus wirtschaftlichen Gründen, aber auch, weil sie in Städten residierten und in mediterran-antiker Tradition von städtischer Mentalität geprägt waren. Kirchenstiftungen blieben nicht auf die Frühzeit beschränkt, sondern wurden das ganze Mittelalter hindurch vollzogen $^{33}$. Dabei ging es nicht immer um die Errichtung ganzer Gotteshäuser, sondern auch um Kapellen oder Altäre, für die besondere Klerikerstellen mit Pfründen geschaffen wurden. Die Stifter waren keineswegs bloß wohlhabende Adlige oder Bürger, vielmehr haben sich in gleicher Weise minder begüterte Menschen zusammengetan, um Stiftungen auf genossenschaftlicher Basis zu errichten. So gab es in den Städten Altäre von Zünften, Gilden und Bruderschaften, und ebenso haben im vorreformatorischen Jahrhundert die Dorfbewohner öfter gemeinsam eine Priesterpfründe oder einen Prediger finanziert ${ }^{34}$. Neben den gutsherrlichen Oratorien, den Kapellen und Pfarrkirchen standen die Klöster, gestiftet für eine größere Anzahl von Mönchen oder Nonnen. Hervorgehoben seien die Stiftskirchen, bei denen das Wort Stiftung sogar namengebend wurde ${ }^{35}$; bei diesen Stiften lebten Kanoniker oder Kanonissen in quasi-monastischer Weise zusammen. Stiftskichen wurden, mindestens seit karolingischer Zeit, ständig gegründet; im Reich zählten sie nach vielen Hunderten. Oft erreichten sie ein bemerkenswertes Alter; so besteht noch heute das Kollegiatstift zur Alten Kapelle in Regensburg, das König Ludwig der Deutsche vor über 1100 Jahren eingerichtet hat ${ }^{36}$. Auch in der mittelalterlichen Doppelstadt Berlin-Cölln hat es ein derartiges Kollegiatstift gegeben; es wurde Mitte des 15. Jahrhunderts durch Kurfürst Friedrich II. an der Kirche des Hohenzollern-Schlosses geschaffen ${ }^{37}$. Dieses sogenannte Domstift der Erasmuskapelle mit insgesamt acht Kanonikern gilt als vornehmste kirchliche Institution unserer Stadt im Mittelalter. 
Der zweite Typ der Stiftungen waren Einrichtungen sozialer Fürsorge. Vor allem die Spitäler in den hoch- und spätmittelalterlichen Städten sind zu nennen, die eine steigende Anzahl von Armen und Bedürftigen aufnahmen ${ }^{38}$; Spitäler dienten aber auch als Herbergen für Reisende oder - in dem uns geläufigen Sinne - als Krankenhäuser. Vorläufer der mittelalterlichen Fürsorgestationen waren die Fremdenhäuser, Armenhäuser, Kranken-, Waisen- und Findelhäuser sowie die Altenheime der christlichen Antike; diese sind im Recht des Kaisers Justinian von 534 nachweisbar und werden nach dem Motiv der frommen Gesinnung bei der Stiftung als ,piae causae“ bezeichnet ${ }^{39}$. Obschon die Spitäler des Mittelalters vielfach von Bürgern, also von Laien, gegründet und getragen wurden, blieben sie in die kirchliche und religiöse Sphäre eingebunden. Stets bildeten nämlich eine Kapelle, Meßfeiern und Gebetszeiten das räumlich-zeitliche Zentrum im Leben der Spitaliten. In Berlin legt davon noch heute eine Backsteinkapelle an der Spandauer Straße Zeugnis ab, die zu dem Ende des 13. Jahrhunderts gestifteten Heiliggeistspital gehört hat ${ }^{40}$.

Kirchen und Spitäler waren Bauwerke, die - aus Stein errichtet eine besondere Affinität zur Stiftung aufwiesen, welche eben auf Dauer angelegt war. Sie waren aber auch Architektur, also Kunstwerke, oder konnten das doch wenigstens sein. Ein Stifter im Mittelalter wurde deshalb leicht zum Mäzen ${ }^{41}$. Vielleicht leuchtet das auf Anhieb eher für die Kirchen ein, die wir noch heute vielerorts bewundern können; man braucht aber bloß nach Lübeck zu reisen und das dortige Spital betrachten, um zu erfahren, daß die gotische Bauweise auch auf dem Gebiet der Spitäler zu Meisterleistungen fähig war. Und selbst die scheinbar so karg und zweckmäßig angelegte Fuggerei in Augsburg aus dem 16. Jahrhundert, eine Wohnsiedlung für verarmte Familien, konnte unlängst noch als Kunstwerk gewürdigt werden ${ }^{42}$. Architektur war eine Ordnungsmacht für andere Künste ${ }^{43}$, und man kann sagen, daß ,nahezu alle im Mittelalter entstandene Kunst (...) aus religiösen Motiven gestiftet" wurde ${ }^{44}$. Tafelmalerei und Glasfenster ${ }^{45}$, Goldschmiedearbeiten und Skulpturen, liturgische Gewänder und Handschriften verdankten ihre Entstehung dem Stifterwillen, und nicht selten wurden Stifterbild, Stifterwappen und Stiftername auf diesen Gegenständen verewigt. 
Schließlich haben Stiftungen Bildung und Wissenschaft gefördert. Das gilt schon für die Schulen, bei denen die Lehrer durch den Genuß einer Pfründe entlohnt werden konnten ${ }^{46}$, besonders aber für die Universitäten. Alle deutschen Universitäten des Mittelalters waren Stiftungsuniversitäten, sie wurden also kaum aus dem Staatshaushalt finanziert ${ }^{47}$. Diese Tatsache wird freilich selbst von modernen Universitätshistorikern verkannt; so wurde kürzlich von der erst 1914 gegründeten Universität Frankfurt am Main fälschlich behauptet, sie sei die einzige Stiftungsuniversität, „die es in der deutschen Geschichte gegeben hat" ${ }^{\text {“48 }}$. An mittelalterlichen Generalstudien bestritten neben den Professoren die bedürftigen Studenten ihren Lebensunterhalt, ihr Stipendium, aus Stiftungsgütern ${ }^{49}$. Wie die anderen Stiftungsinstitutionen unterlagen die Universitäten von Anfang an einer religiösen Bestimmung. Die Gründungsurkunden verraten, daß die hohen Schulen dem Lobe Gottes, der Verbreitung des Glaubens und der Christenheit zum Trost dienen sollten, und dementsprechend war der Lebensrhythmus der Doktoren, Magister und Scholaren tiefgreifend kirchlich geprägt.

Stiftungen des Mittelalters waren, wie sich gezeigt hat, Phänomene, in denen sich Religiöses, Rechtliches und Ökonomisches durchdrang und gegenseitig bedingte, und in den je konkreten Ausprägungen kamen die Motive der Caritas oder des Mäzenatentums hinzu. Was in unserer Aufzählung noch fehlt, ist der Begriff des Sozialen. Für Mauss stand das Soziale im Zentrum der von ihm beschriebenen totalen Phänomene, und tatsächlich erweist sich auch an den Stiftungen, daß es ein sozialer Mechanismus war, der sie in Gang gesetzt und in Gang gehalten hat. Einem solchen Verständnis der Stiftung hat die Forschung allerdings erst in den letzten Jahren den Weg gewiesen. Maßgeblich wurde dabei die Erkenntnis, daß Stiftungen ursprünglich immer zugleich Totenstiftungen waren ${ }^{50}$. Sie dienten also zur Versorgung der Stiftergräber oder zur Wahrung der Stiftermemoria. Stiftungen sind dadurch ausgezeichnet, daß sie in scheinbar altruistischer Weise einem gesellschaftlichen oder kulturellen Mangel abhelfen wollen, daneben aber dem Fortleben des Stifters und seines Namens gewidmet sind. Schon in der vorchristlichen Antike ist der Zusammenhang deutlich faßbar. So hat der griechische 
Philosoph Epikur, der im Jahr 270 vor unserer Zeitrechnung starb, durch eine testamentarisch festgelegte Stiftung den Bestand seiner Schule gesichert ${ }^{51}$; Epikur verpflichtete gleichzeitig seine Schüler zu einem aufwendigen Erinnerungskult. Aus den Einkünften der Stiftung sollten nämlich seinen Eltern, seinem Bruder und ihm selbst regelmäßige Totenopfer dargebracht werden, und an jedem Zwanzigsten eines Monats waren die Schüler zu einem Totenmahl im Gedenken an Epikur verpflichtet. Tatsächlich scheinen die Vorschriften des Vermächtnisses rund 500 Jahre beachtet worden zu sein; schon Plutarch hat nach eigenem Zeugnis an den Totenmählern der Epikuräer teilgenommen.

Im Mittelalter und unter christlichen Vorzeichen konnte es selbstverständlich derartige Totenkultstiftungen nicht mehr geben; an ihre Stelle traten, wie Karl Schmid formuliert hat, Stiftungen für das Seelenheil ${ }^{52}$. Die Stiftungen sollten dazu verhelfen, die Seele beim Jüngsten Gericht zu retten. Adressat der Seelenheilstiftungen war eigentlich Gott selber, der für die gute Tat das ewige Leben geben konnte ${ }^{53}$. Im übrigen erhoffte der Stifter die dauernde Fürbitte der von ihm geförderten Menschen. Gebet und Memoria waren die ständige Gegengabe für die Gabe des Stifters ${ }^{54}$. Zu diesen Leistungen waren die Priester, Altaristen, Mönche und Stiftsherren ebenso verpflichtet, wie die Spitalinsassen, Professoren und Studenten. Die Künstler bewirkten die Stiftermemoria durch Inschrift oder Bild. Die Stiftung, die einen wirklichen Gabentausch im Sinne von Mauss begründete, rief wegen ihres dauernden Bestandes eine ständige Interaktion zwischen Lebenden und Toten hervor. Der tote Stifter als Spender der Wohltaten und als Nutznießer der ihm gewidmeten Gebete wurde als reale Person aufgefaßt. Sinnfällig wurde das beispielsweise dann, wenn bei Totenmählern dem Kommemorierten selbst Speisen geopfert wurden $^{55}$. Durch die Stiftung gewann der Verstorbene wieder Gegenwart unter den Lebenden ${ }^{56}$; nach Auffassung der Zeit war er es selbst, der die Erträge seiner Stiftung als fromme Gaben verteilte. Die Stiftungen beruhten auf einer Denkform, die in der europäischen Geschichte erst während der Zeit Napoleons und Goethes aufgegeben wurde: auf der Vorstellung nämlich, daß Lebende und Tote gemeinsam die Gesellschaft bilden.

Wenn das Konzept der totalen sozialen Phänomene durch Mar- 
cel Mauss irgendeinen Mangel aufweist, dann wohl den, daß in ihm das Politische eine eher untergeordnete Rolle spielte ${ }^{57}$. Diese Akzentsetzung kam seinerzeit den Intentionen der französischen Historiker entgegen. Wenn wir aber heute erörtern wollen, ob die Stiftungen das Zentrum im Bezugssystem einer totalen Geschichte sein könnten, dürfen wir diesen wichtigen Aspekt nicht beiseitelassen. Als Beispiel für den Stellenwert der Politik im Stiftungswesen soll im folgenden das Wirken des bayerischen Herzogs Ludwigs des Gebarteten dienen. Die Stiftungen dieses Wittelsbachers zeigen im übrigen ein so komplexes Bedingungsgefüge, daß sie nahezu den Idealtyp eines totalen sozialen Phänomens repräsentieren.

Der Herzog hat seine Regierung im Jahr 1416 angetreten $^{58}$; Ludwig der Bärtige war aber keineswegs der Gesamtherrscher in Bayern, sondern nur einer von vier Wittelsbachern, die den Herzogstitel führten. Von seinem Vater hatte Ludwig ein äußerst zersplittertes Territorium geerbt, das in Ingolstadt seine Hauptstadt hatte. Trotzdem gelang es Ludwig und seinen Nachfolgern, im Teilherzogtum Bayern-Ingolstadt während des 15. Jahrhunderts einen modernen Territorialstaat zu errichten, der den anderen wittelsbachischen Herrschaften kaum nachstand ${ }^{59}$. Ludwig selbst konnte sich intensive Erfahrungen aus Frankreich zunutze machen, da er als Schwager Karls VI. jahrzehntelang am Pariser Königshof gelebt hatte ${ }^{60}$. Beim Aufbau eines rational organisierten Staatsgebildes ließ er sich durch zahlreiche Männer unterstützen, die ihm schon in Paris gedient hatten; diese besetzten in Ingolstadt die einflußreichsten Hofämter und die wichtigsten Stellungen in Rat und Kanzlei. Der Zentralismus des Herzogs ging selbstverständlich zu Lasten anderer Herrschaftsträger, und auch die politischen Freiheiten der Bürgerschaften schränkte Ludwig ein. Dafür förderte er die Wirtschaftskraft und bauliche Entwicklung seiner Residenz. Das erste große Projekt wurde der Neubau einer herzoglichen Feste; dieses „Neue Schloß“ vereinigte die Funktionen des Wehrbaus, des Wohn- und des Regierungssitzes in sich $^{61}$. Als Pendant zu dem herrschaftlichen Bauwerk entstand unter Ludwig auf der anderen Seite der Stadt die Liebfrauenkirche $^{62}$. Schon Ludwigs Vater hatte den Ingolstädtern eine Marienkirche als zweite Pfarrkirche gestiftet, die jetzt sein Sohn als 
großartige Hallenkirche aufführen lie $\beta^{63}$. Von Beginn an hat zwischen Kirche und Hof eine enge Verbindung bestanden; denn die Herzöge hatten sich das Präsentationsrecht vorbehalten und die Pfründe des Pfarrherrn an ihre Kanzleischreiber oder Räte vergeben. Der erste Frauenpfarrer war ein gelehrter Jurist, der zweite ein Artistenmagister, Theologe und Mediziner, der gar in Paris als Universitätsprofessor gelehrt hatte und nun Ludwig dem Bärtigen als Leibarzt diente ${ }^{64}$. Mit dem Jahr 1429 traten aber die Beziehungen Herzogs Ludwigs zur Frauenkirche in ein ganz neues Stadium; der Wittelsbacher bestimmte nämlich die zweite Ingolstädter Pfarrkirche zur Grablege für sich und seine Familie ${ }^{65}$. 1430 ließ er schon den Leichnam seines Vaters und das Herz seiner ersten Gemahlin von anderswoher in die Gruft der Frauenkirche überführen; und wohl im selben Jahr hat ein Ulmer Künstler nach Anweisungen Ludwigs das Modell für dessen eigenes Grabmal geschaffen ${ }^{66}$. In der darauffolgenden Zeit wurde die ganze Frauenkirche im Hinblick auf die Grab- und Gedächtnisstiftung sachlich und personell überaus reich ausgestattet ${ }^{67}$. Nicht ohne Grund hat man von der Stiftung gesprochen als einem „Gesamtkunstwerk von höchstem thematischen und künstlerischen Anspruch" ${ }^{\text {“68 }}$. Zur Ausstattung der Kirche gehörten neben silbernen Altarbildern und einem Figurenschmuck aus Stein, neben liturgischen Geräten, Ornaten und Büchern auch ein Glasfensterzyklus, auf dem die Ingolstädter Linie der Wittelsbacher dargestellt war. Ein Schatz von Reliquien und Kleinodien, z.T. aus Frankreich, wurde dem Gotteshaus übereignet. Besonderen Wert legte Ludwig auf die polyphone und instrumentale Kirchenmusik. So stellte er erhebliche Mittel für einen Organisten bereit. Die Mitte des Stiftungswerkes bildeten aber die von Ludwig kreierten Personengemeinschaften, die das Gebetsgedenken vollziehen sollten. Eine Gruppe von sechs Kaplänen sollte zusammen mit dem Pfarrer täglich eine Reihe von Gedenkmessen mit unterschiedlicher Feierlichkeit zelebrieren ${ }^{69}$. An diesen beteiligt waren der Orgelspieler und verschiedene Sangmeister mit ihren Chören, die sich aus Schülern und Psalteristen zusammensetzten. Die aus 16 Personen bestehende Gruppe der Psalteristen hatte außerdem die Aufgabe, in Vierergruppen wechselnd ohne Unterlaß den Psalter zu beten ${ }^{70}$. Auch die Armen erhielten ihren Platz in der 
Commemoratio. Der Herzog stiftete dazu ein neues Spital neben der Liebfrauenkirche, in dem 15 Pfründner wohnen sollten ${ }^{71}$. Der Tagesablauf der Spitaliten war ausschließlich auf die Gebetsleistungen abgestellt. Sie hatten an den Gottesdiensten in der Kirche und in der Kapelle ihres eigenen Hauses teilzunehmen, bei den sieben Tagzeiten insgesamt 206 Paternoster und Ave Maria zu sprechen und auch bei den Mahlzeiten des Stifters zu gedenken. Grabsorge und Gebetsgedächtnis in der Marienkirche sollten allerdings nicht auf Ludwig den Bärtigen selbst beschränkt bleiben. Vielmehr schloß der Herzog in seine Memorialvorschriften immer wieder seine Angehörigen mit ein. Er griff dabei bis zu seinem wittelsbachischen Spitzenahn, Kaiser Ludwig dem Bayern, zurück und nannte dazu seine unmittelbaren Vorfahren und Angehörigen ${ }^{72}$. Schließlich wollte er die Heilswirkung der Gebete noch allen Dienern und Untertanen sowie allen Gläubigen schlechthin zuwenden ${ }^{73}$. In der Gedächtnisstiftung hat sich, wie man sagen kann, der werdende Territorialstaat in seinem Fürstenhaus sakral selbst dargestellit ${ }^{74}$. Wohl am eindrucksvollsten zeigt sich das Zusammenspiel von Stiftung, Gedenken und Politik an dem sogenannten Fürstenjahrtag ${ }^{75}$. Die Einrichtung ging in Bayern bereits auf Kaiser Ludwig, also auf die erste Hälfte des 14. Jahrhunderts, zurück. Danach waren die Weltgeistlichen sämtlicher Dekaneibezirke verpflichtet, alljährlich am St. Blasius-Tag zu einem zentralen Ort des Herzogtums zu kommen, um an einem zweitägigen Anniversar für des Kaisers Vorfahren teilzunehmen. Als Gegenleistung hatte Ludwig der Bayer der Geistlichkeit die Testierfreiheit gewährt. Ludwig der Bärtige übertrug nun den Jahrtag an die Ingolstädter Liebfrauenkirche. Jedes Jahr am 4. Sonntag nach Pfingsten sollten rund 160 bepfründete Weltpriester zum Totengedenken nach Ingolstadt kommen ${ }^{76}$. Wenn man weiß, welche Schlüsselrolle die Pfarrherrn der Zeit bei der Verbreitung von Nachrichten spielten, kann man ermessen, daß der Fürstenjahrtag zur Stabilisierung politischer Herrschaft taug$\mathrm{te}^{77}$.

Der großangelegte Stiftungsplan Ludwigs des Bärtigen ${ }^{78}$ wurde allerdings nur in bescheidenem Umfang realisiert. Der Herzog hatte sich gegen Ende seines Lebens mit seinem einzigen legitimen Sohn überworfen, der dann auch noch vor ihm starb. Lud- 
wig selbst verschied im Exil (1447) und wurde niemals, wie es sein Wunsch gewesen war, nach Ingolstadt überführt. Der Herzogsgruft fehlte also ihr Zentrum, und das Grabmonument blieb unausgeführt. Ingolstadt fiel an eine andere Wittelsbacher Linie, die an Ludwigs Werk kaum interessiert sein konnte ${ }^{79}$. Ohne Rückhalt an der politischen Herrschaft konnte die Stiftung nicht gedeihen; immerhin war soviel Kapital angehäuft, daß dafür eine neue Verwendung gefunden werden mußte. So entstand, nur ein Vierteljahrhundert nach Herzog Ludwigs Tod, die Universität Ingolstadt, die Vorgängerin der gegenwärtig größten deutschen Universität in München ${ }^{80}$.

Stiftungen wie diejenige des Herzogs von Ingolstadt führen wie von selbst auf die Frage, inwiefern sie als totale soziale Phänomene Knotenpunkte oder Leitmotive einer totalen Geschichte sein könnten. Zweifellos haben sie eine große Zahl von Menschen und Institutionen bestimmt, aber konnten sie auch eine Gesellschaft insgesamt in Gang halten, wie dies Mauss bei den totalen Tatsachen für möglich hielt? Der Forschungsstand läßt nicht zu, darauf heute eine klare Antwort zu geben. Daß immerhin die Frage so gestellt werden kann, rechtfertigt die Kritik, in die das Stiftungswesen in der Reformationszeit und besonders während der Aufklärung geraten ist ${ }^{81}$. Ein ähnliches Argument liefern die islamischen Länder. Hier soll bis ins 20. Jahrhundert hinein ein Viertel bis über die Hälfte des Volksvermögens in Stiftungen angelegt gewesen sein; Modernisten wie Atatürk oder die Pahlewis sahen sich deshalb zu Gegenmaßnahmen veranlaßt ${ }^{82}$. Und wenn es richtig ist, daß die Stiftungen in der Vormoderne die Gesellschaft der Lebenden und Toten zu organisieren halfen, fällt es schwer, ihnen einen geringeren Stellenwert zuzuschreiben, als anderen sozialen Ordnungsprinzipien, als Herrschaft beispielsweise oder als Genossenschaft ${ }^{83}$. Aber zweifellos wissen wir noch sehr wenige Einzelheiten darüber, welche Bedeutung den Stiftungen im gesamtgesellschaftlichen Gefüge zugekommen ist. Am weitesten konnte in dieser Hinsicht bisher wohl die stadtgeschichtliche Forschung vorstoßen. In einer gesellschaftsgeschichtlichen Strukturanalyse Augsburgs im Spätmittelalter wurde z.B. gezeigt, daß sich das alle Lebensbereiche prägende 
Verhältnis zwischen Bürgerschaft und Kirche signifikant am Stiftungsverhalten ablesen läßt ${ }^{84}$. Je nach sozialem Rang und nach der Gruppenzugehörigkeit wählte man demnach für seine Stiftungen die Kirchen und Klöster aus, an denen man im Gegenzug seinen nachgeborenen Söhnen und Töchtern Unterkunft und Pfründe zu sichern verstand. Durch eine andere Studie wurde demonstriert, wie sich die Stiftungen im Rahmen zweier fränkischer Kleinstädte durch die Jahrhunderte behaupteten bzw. in ihrer Erscheinungsweise wandelten ${ }^{85}$. Weitere Untersuchungen dieser Art, bezogen freilich auch auf Adelsherrschaften und Territorien, sind künftig notwendig - und erfolgversprechend. Ein anderer Ansatz, der mit Sicht auf die totale Geschichte zu verfolgen sein wird, betrifft das Problem der Periodisierung. Bisher wissen wir zwar, daß das Stiftungswesen in der Geschichte immer wieder aufgekommen ist, daß es aber allem Anschein nach keine kulturelle Konstante dargestellt hat. In Griechenland vor dem Hellenismus, in Rom vor ca. 100 n.Chr. ${ }^{86}$ und in den ersten Jahrhunderten des Mittelalters hat es offenbar gefehlt oder nur in Kümmerformen existiert $^{87}$. Befriedigende Erklärungen für derartige Zeitenwechsel liegen bis jetzt nicht $\operatorname{vor}^{88}$, zumal eine moderne historische Gesamtdarstellung des Stiftungswesens fehlt ${ }^{89}$. In der neueren Geschichte scheint eine Deutung leichter zu sein, weiß man doch, daß Stiftungen in autoritären Staaten nicht gedeihen können ${ }^{90}$. Wo der Staat die gesellschaftlichen Bedürfnisse definiert, haben die Stifter keine Entfaltungschance. Bekanntlich hat deshalb auch die DDR die Stiftungen nicht gefördert ${ }^{91}$; im Zivilgesetzbuch von 1975 kamen sie gar nicht mehr vor ${ }^{92}$.

Das Postulat der totalen Geschichte richtet sich ebenso wie auf die Vergangenheit auf die Gegenwart. Was können wir also über uns und unsere Zeit erfahren, wenn wir die Stiftungen des Mittelalters studieren? Es ist klar, daß in den Versuch meiner Antwort auf diese Frage eigene Beweggründe zu dem Forschungsthema eingehen. Zunächst scheint mir evident zu sein, daß wir in einer Gesellschaft leben, in der der Staat nur einen Bruchteil dessen erfüllen kann, was erforderlich wäre. Vielleicht niemals zuvor seit 45 Jahren war private Initiative zur Lösung öffentlicher Aufgaben mehr geboten als jetzt, vielleicht gab es aber dafür auch nie größere Handlungsräume. Selbstverständlich kön- 
nen und sollen wir nicht alle Stifter werden, aber die Sensibilität für die Not der anderen und für das Neue, dem eine Chance gegeben werden muß, können wir aus der Geschichte lernen. Die Geschichte der Stiftungen übertreibt freilich die Motive der Fürsorge und des Mäzenatentums nicht; sie zeigt im Gegenteil, wieviel Selbstsucht den edlen Absichten beigemischt ist, sie ist deshalb ehrlich und menschlich. Schließlich ein Letztes: Der Stifter und der Historiker sind aufs engste miteinander verwandt. Für beide steht im Zentrum ihres Denkens und Handelns das Thema der Dauer. Der Historiker will die Vergangenheit vor dem Vergessen bewahren und kann damit leidlich erfolgreich werden; als Geschichtsschreiber wollte er bis vor kurzem auch in seinem Werk überleben. Diese Illusion hat uns Max Weber genommen. Geschichtswissenschaft ist - um mit Wolfgang Hardtwig zu sprechen - keine Geschichtsreligion mehr, sondern sie ist Forschung geworden $^{93}$; jede Erkenntnis bezweckt ihre eigene Revision. Ganz ähnlich steht es mit dem Stifter. All seine Energie, Dauerndes zu schaffen, bleibt letztlich vergeblich, wenn es auch nicht so schnell gehen muß, wie bei Herzog Ludwig von Ingolstadt. Das Studium der Stiftungen kann deshalb dazu verhelfen, das Maß zu finden, das dem Menschen in seiner Geschichte gegeben ist. 


\section{Anmerkungen}

1 Marcel Mauss, Essai sur le don, Paris 1950; dt. Übers. von Eva Moldenhauer: Die Gabe. Form und Funktion des Austauschs in archaischen Gesellschaften. Mit einem Vorwort von E.E. Evans-Pritchard, Frankfurt am Main 1984.

2 Ebd. (dt. Ausgabe) S. 17f.; zu den folgenden Aussagen im Text vgl. S. 36f., 91-103; 33, 35f.; 21f.

3 Ebd. (dt. Ausgabe) S. 17f., 90, 176.

4 Ebd. S. 178.

5 Ebd. S. 19, 163.

6 Ebd. S. 161.

7 Ebd. S. 160 (auch das folgende Zitat). Zur innenpolitischen Lage Frankreichs in der Mitte der zwanziger Jahre vgl. Rudolfvon Albertini, Frankreich vom Frieden von Versailles bis zum Ende der Vierten Republik 1919-1958, in: Handbuch der europäischen Geschichte 7.1, hg. von Theodor Schieder, Stuttgart 1979, S. 438-444, hier bes. S. 442.

8 Mauss (wie Anm. 1, dt. Ausgabe) S. 162f.

9 Jacques Le Goff-Roger Chartier - Jacques Revel, Le nouvelle histoire, Paris 1978; bes. J. Le Goff, L'histoire nouvelle, S. 210-241, mit Bezug auf Mauss: S. 230. Ebd. der prosopographische Artikel über Marcel Mauss S. 394-398. Vgl. auch neuerdings die forschungsgeschichtlichen Arbeiten von Sabine Jöckel, „Nouvelle histoire“ und Literaturwissenschaft, 2 Bde., Rheinfelden 1985, bes. I S. 30-32, und von Annette Rieks, Französische Sozial- und Mentalitätsgeschichte. Ein Forschungsbericht, Altenberge 1989, S. 20, 41, 64.

10 Jacques Le Goff - Pierre Toubert, Une histoire totale du Moyen Age estelle possible?, in: Tendances, perspectives et méthodes de l'histoire médiévale (Actes du $100^{\mathrm{e}}$ congrès national des sociétés savantes, Paris 1975, Section de philologie et d'histoire jusqu'à 1610, Tome I), Paris 1977, S. 31-44, hier S. 33.

11 Das Aufkommen des Begriffes ist nicht näher untersucht, doch scheint er auf Fernand Braudel zurückzugehen: J.H. Hexter, Fernand Braudel and the Monde Braudellien ..., in: Journal of Modern History 44 (1972), S. 480-539, hier S. 511. Braudel verwandte gleichzeitig die Begriffe ,histoire globale" und - im Hinblick auf Lucien Febvre - „histoire à part entière": Note liminaire zu: Lucien Febvre, Pour une Histoire à part entière, Paris 1962. Vgl. Dieter Groh, Strukturgeschichte als „totale“ Geschichte?, in: Vierteljahrschrift für Sozial- und Wirtschaftsgeschichte 58 (1971), S. 289-322, hier S. 310; Karl-Georg Faber, Geschichtslandschaft - Région historique - Section in History. Ein Beitrag zur verglei- 
chenden Wissenschaftsgeschichte, in: Saeculum 30 (1979), S. 4-21, hier S. 19-21 (mit Hinweis auf Pierre Goubert und Emmanuel Le Roy Ladurie); Johannes-Michael Scholz, Historische Rechtshistorie. Reflexionen anhand französischer Historik, in: Vorstudien zur Rechtshistorik, hg. von Dems. (Ius Commune, Sonderhefte 6), Frankfurt am Main 1977, S. 1-175, hier bes. S. 38f. (mit zusätzlichem Hinweis auf die marxistischen Annalesisten Vilar und Lefebvre).

12 Hexter (wie Anm. 11) S. 511 (unter Bezug auf ein Zitat Braudels). Vgl. Jöckel (wie Anm. 9) I S. 69, 73, 76, Rieks (wie Anm. 9) S. 65. - In dem programmatischen Aufsatz von Le Goff und Toubert von 1975/1977 (wie Anm. 10) fehlt ein Hinweis auf Mauss.

13 Jacques Le Goff, Der Appetit auf Geschichte, in: Pierre Chaunu - Georges Duby - J. Le Goff - Michelle Perrot, Leben mit der Geschichte. Vier Selbstbeschreibungen, hg. von Pierre Nora, aus dem Französischen von Eva Moldenhauer, Frankfurt am Main 1989, S. 100-177, hier S. 164. (Im französischen Original: Essais d'ego-histoire, Paris 1987, S. 173239, hier S. 226). Vgl. Otto Gerhard Oexle, Das Andere, die Unterschiede, das Ganze. Jacques Le Goffs Bild des europäischen Mittelalters, in: Francia 17/1 (1990), S. 141-158, hier bes. S. 141.

14 Zur „histoire problème“ "Lucien Febvres: Rieks (wie Anm. 9) S. 13, 30, 66; zu Braudel: Hexter (wie Anm. 11) S. 510f.

15 Otto Gerhard Oexle, Die Geschichtswissenschaft im Zeichen des Historismus. Bemerkungen zum Standort der Geschichtsforschung, in: Historische Zeitschrift 238 (1984), S. 17-55; Ders., „Historismus“. Überlegungen zur Geschichte des Phänomens und des Begriffs, in: Braunschweigische Wissenschaftliche Gesellschaft, Jahrbuch 1986, S.119155; Wolfgang Hardtwig, Geschichtsreligion - Wissenschaft als Arbeit - Objektivität. Der Historismus in neuer Sicht, in: Historische Zeitschrift 252 (1991), S. 1-32, hier S. 18f.

16 Ernst Schulin, Geschichtswissenschaft in unserem Jahrhundert. Probleme und Umrisse einer Geschichte der Historie (Schriften des Historischen Kollegs, Vorträge 16), München 1988, S. 26f. Vgl. dazu den Aufsatz von Le Goff - Toubert von 1975/77 (wie Anm. 10) und die Publikation des Sammelwerkes La nouvelle histoire von 1978 (oben Anm. 9).

17 Für die Mediävistik nur zwei Beispiele aus sehr verschiedenen Gegenstandsbereichen: František Graus, Pest - Geissler - Judenmorde. Das 14. Jahrhundert als Krisenzeit (Veröffentlichungen des Max-Planck-Instituts für Geschichte 86), Göttingen 1987, S. 8; Theo Kölzer, Studien zu den Urkundenfälschungen des Klosters St. Maximin vor Trier (10.-12. Jahrhundert) (Vorträge und Forschungen, Sonderband 36), Sigmaringen 1989, S. 312. Für die neuere Sozialgeschichte: Jürgen Kocka, Sozial- 
geschichte. Begriff - Entwicklung - Probleme, Göttingen ² 1968, S. 100, 210.

19 Johannes Fried, Die Formierung Europas 840-1046 (Oldenbourg Grundriß der Geschichte, Bd. 6), München 1991, S. 111.

20 Hans-Ulrich Wehler, Deutsche Gesellschaftsgeschichte, Erster Band: Vom Feudalismus des Alten Reiches bis zur Defensiven Modernisierung der Reformära 1700-1815, München 1987, ${ }^{2} 1989$, S. 7.

21 Oexle (wie Anm. 13) S. 142.

22 Vgl. Dens., „Der Teil und das Ganze“ als Problem geschichtswissenschaftlicher Erkenntnis. Ein historisch-typologischer Versuch, in: Teil und Ganzes. Zum Verhältnis von Einzel- und Gesamtanalyse in Geschichts- und Sozialwissenschaften, hgg. von Karl Acham - Winfried Schulze (Theorie der Geschichte. Beiträge zur Historik, Bd. 6), München 1990, S. 348-384, hier S. 381.

23 So haben sich Le Goff und Toubert, auf der Suche nach der Formierungsphase einer „histoire totale“, selbst u.a. auf Ranke berufen: Wie Anm. 10, S. 31f. - Mißverständlich (im Sinne einer positivistischen Geschichtsauffassung) hat sich beispielsweise Pierre Goubert geäußert (Beauvais et le Beauvaisis de 1600 à 1730. Contribution à l'histoire sociale de la France du XVII ${ }^{\mathrm{e}}$ siècle, T. I, Paris 1960, ND Paris 1982, S. VIII): „Avec un enthousiasme de néophyte, j'ai voulu d'abord retrouver le reflet, le témoignage du $\mathrm{XVII}^{\mathrm{e}}$ siècle 'total' dans une province modeste“. S.a. Scholz (wie Anm. 11) S. 39 mit einem Zitat von Pierre Chaunu: „La historia total ... Es un deseo, marca una dirección ... una reacción contra una historia que se juzgaba excesivamente polarizada por la política y el Estado“. Ähnlich Chaunu auch in: Leben mit der Geschichte (wie Anm. 13) S. 34. - Le Goff (ebd., S. 132f.; 158) rückt neuerdings von der „histoire totale“ ab, und zwar mit Akzenten, die wiederum Fehlinterpretationen begünstigen: „Nie hat mich eine Geschichte zu interessieren vermocht, die sich auf einen eingegrenzten Sachverhalt beschränkt. Gewiß bedarf es der Verankerung in den Einzelheiten, weil man nichts wirklich Aufschlußreiches entdecken kann, solange man nicht mit den Einzelheiten vertraut ist. Aber man muß in Zusammenhängen denken. Ich sehe keine wahrhafte Geschichte, die sich nicht vornimmt, gleichsam Weltgeschichte zu sein - heute würde ich eher sagen: eine allgemeine, die das Maximum an möglicher Geschichte berücksichtigt, ausgehend von derjenigen, die man am besten kennt (...). Die Parole einer globalen oder einer totalen Geschichte ist nützlich und notwendig gewesen, und wir müssen diesen Horizont im Auge behalten. Aber ich frage, ob es nicht lohnender wäre, mit einer wirklich vergleichenden Geschichte zu beginnen, nicht um zu einer globalen, son- 
dern zu einer - wie ich Foucault einige Monate vor seinem Tod sagen hörte - allgemeinen Geschichte [histoire générale] zu gelangen“.

24 Otto Gerhard Oexle, Das Evangeliar Heinrichs des Löwen als geschichtliches Denkmal, in: Das Evangeliar Heinrichs des Löwen. Kommentar zum Faksimile, hg. von Dietrich Kötzsche, Frankfurt am Main 1989, S. 9-27, hier S. 19; vgl.: Ders., Memoria und Memorialbild, in: Memoria. Der geschichtliche Zeugniswert des liturgischen Gedenkens im Mittelalter, hgg. von Karl Schmid - Joachim Wollasch (Münstersche Mittelalter-Schriften, Bd. 48), München 1984, S. 384-440, hier bes. S. 394; Ders., Memoria und Memorialüberlieferung im früheren Mittelalter, in: Frühmittelalterliche Studien 10 (1976), S. 70-95, S. 87.

$25 \mathrm{Vgl}$. den neueren Überblick bei Reiner Schulze, Art. Stiftungsrecht, in: Handwörterbuch zur deutschen Rechtsgeschichte, Bd. IV (32. Lief.), Berlin 1990, Sp. 1980-1990. Nicht recht befriedigend ist aus historischer Sicht das Buch von Hans Liermann, Handbuch des Stiftungsrechts, I. Bd.: Geschichte des Stiftungsrechts, Tübingen 1963.

26 Mohammed Rassem, Die Idee der Stiftung im Islam, in: DUZ Universitätszeitung 43.1-2 (1987), S. 16f.; Handbuch des Stiftungsrechts, hg. von Werner Seifart, München 1987, S. 51, mit Lit. in Anm. 1 (Hermann Coing).

27 Bernhard Laum, Stiftungen in der griechischen und römischen Antike. Ein Beitrag zur antiken Kulturgeschichte, 2 Bd., Leipzig - Berlin 1914; vgl. Michael Borgolte, Die Stiftungen des Mittelalters in rechts- und sozialhistorischer Sicht, in: Zeitschrift der Savigny-Stiftung für Rechtsgeschichte 105, Kan. Abt. 74 (1988), S. 71-93, bes. S. 76 (mit Lit.).

28 Johannes von den Driesch, Geschichte der Wohltätigkeit, Bd. I: Die Wohltätigkeit im alten Ägypten, Paderborn 1959, S. 170.

29 Vgl. Rassem (wie Anm. 25) S. 16 (mit Hinweis bes. auf Frankreich: Kein Stiftungsrecht im Code Napoléon; Einfluß des Juristen Maurice Hauriou auf die Entwicklung eines rechtssoziologischen Begriffes der fondation); zu der englisch-amerikanischen Philantropie, die ihre Wurzeln in der englischen Reformation habe, Klaus Neuhoff, Amerikanische Stiftungen. Organisation, Kapitalverhältnisse und Arbeitsweise, Baden-Baden 1968, bes. S. 28-38.

30 Borgolte (wie Anm. 26).

31 Handbuch des Stiftungsrechts (wie Anm. 25) S. 2: Die Stiftung ist „das Ergebnis eines als 'Stiften' bezeichneten Vorgangs, der von einem Stifter ausgelöst wird und von einem Stifterwillen getragen ist (...). Im einzelnen setzt eine Stiftung voraus, daß ein Stifter (oder eine Mehrzahl von Stiftern) in einem Stiftungsgeschäft förmlich den Willen bekundet, zur Verwirklichung eines bestimmten Zwecks auf Dauer eine Stiftung 
zu errichten und diese mit den hierzu benötigten Mitteln (Vermögen) und einer zweckentsprechenden Organisation (Vorstand) auszustatten. Der Stiftungszweck, das Stiftungsvermögen und die Stiftungsorganisation sind also die drei wesentlichen den Stiftungsbegriff prägenden Elemente".

32 Mauss (wie Anm. 1) S. 90, 177.

33 Michael Borgolte, Die mittelalterliche Kirche (Enzyklopädie Deutscher Geschichte, Bd. 17), München 1992, S. 35.

34 Auf das Problem von Eigenkirchen- bzw. Patronatsrecht gehe ich hier nicht ein. Vgl. aber Borgolte (wie Anm. 32) S. 35f., 101-103; Ders., Stiftergrab und Eigenkirche. Ein Begriffspaar der Mittelalterarchäologie in historischer Kritik, in: Zeitschrift für Archäologie des Mittelalters 13 (1985), S. 27-38.

35 Borgolte (wie Anm. 32) S. 104 (mit Lit.).

36 Ebd. S. 48-52.

37 Das Beispiel bei Peter Moraw, Hessische Stiftskirchen im Mittelalter, in: Archiv für Diplomatik 23 (1977), S. 425-458, hier S. 430.

38 Dietrich Kurze, Die Kirche, in: Bürger, Bauer, Edelmann. Berlin im Mittelalter, Berlin 1987, S. 130-162, S. 146f.

39 Jüngere Monographien: Ulrich Knefelkamp, Das Heilig-Geist-Spital in Nürnberg vom 14.-17. Jahrhundert. Geschichte, Struktur, Alltag, Nürnberg 1989; Ders., Stiftungen und Haushaltsführung im Heilig-Geist-Spital in Nürnberg, 14.-17. Jahrhundert, Bamberg 1989; AnnetteBoldt, Das Fürsorgewesen der Stadt Braunschweig in Spätmittelalter und Früher Neuzeit. Eine exemplarische Untersuchung am Beispiel des St. ThomaeHospitals, Braunschweig 1988.

40 Hans-Rudolf Hagemann, Die Stellung der Piae Causae nach justinianischem Rechte, Basel 1953; Borgolte (wie Anm. 26) S. 81f.

41 Kurze (wie Anm. 37) S. 142f., 146.

42 Zum Mäzenatentum vgl.: Artistes, artisans et production artistique au moyen âge. Colloque international, Centre National de la Recherche Scientifique, Université de Rennes II - Haute-Bretagne, 2-6 mai 1983, organisé et édité par XaverBarral i Altet, Vol. II: Commande et travail, Paris 1987; Corine Schleif, Donatio et Memoria. Stifter, Stiftungen und Motivationen an Beispielen aus der Lorenzkirche in Nürnberg, München 1990; Wolfgang Schmid, Kunststiftungen im spätmittelalterlichen Köln, in: Materielle Kultur und religiöse Stiftung im Spätmittelalter (Österreichische Akademie der Wissenschaften, Philosophisch-historische Klasse, Sitzungsberichte, 554. Band), Wien 1990, S. 157-185; Peter Hirschfeld, Mäzene. Die Rolle des Auftraggebers in der Kunst, o.O. 1968. - Joachim Bumke, Mäzene im Mittelalter. Die Gönner und Auf- 
traggeber der höfischen Literatur in Deutschland 1150-1300, München 1979; Ders. (Hg.), Literarisches Mäzenatentum. Ausgewählte Forschungen zur Rolle des Gönners und Auftraggebers in der mittelalterlichen Literatur (Wege der Forschung 598), Darmstadt 1982. - Weitere Literaturhinweise im Artikel von Michael Mollat, Mäzen, Mäzenatentum, in: Lexikon des Mittelalters, Band VI.2 (1992), Sp. 430-433.

43 Marion Tietz-Strödel, Die Fuggerei in Augsburg. Studien zur Entwicklung des sozialen Stiftungsbaus im 15. und 16. Jahrhundert, Tübingen 1982.

44 Ebd. S. 217 (nach H. Sedlmayr).

45 Elisabeth Heller, Das altniederländische Stifterbild (tuduv Studien, Reihe Kunstwissenschaften, Bd. 6), München 1976, S. 1.

46 Neben Heller (wie Anm. 44) und der Anm. 41 zit. kunstgeschichtlichen Literatur vgl. z.B.: Dirk Kocks, Die Stifterdarstellung in der italienischen Malerei des 13.-15. Jahrhunderts, Diss. Köln 1971; ElisabethVavra, Pro remedio animae - Motivation oder leere Formel. Überlegungen zur Stiftung religiöser Kunstobjekte, in: Materielle Kultur und religiöse Stiftung (wie Anm. 41) S. 123-156; Vitrea dedicata. Das Stifterbild in der deutschen Glasmalerei des Mittelalters, hrsg. von der Stiftung Volkswagenwerk Hannover, Berlin 1975.

47 Vgl. Martin Kintzinger, Das Bildungswesen in der Stadt Braunschweig im hohen und späten Mittelalter. Verfassungs- und institutionengeschichtliche Studien zu Schulpolitik und Bildungsförderung (Beihefte zum Archiv für Kulturgeschichte 32), Köln - Wien 1990, bes. S. 359366.

48 Michael Borgolte, Stiftungen des Mittelalters im Spannungsfeld von Herrschaft und Genossenschaft, in: Memoria in der Gesellschaft des Mittelalters, hgg. von Dieter Geuenich - Otto Gerhard Oexle, im Druck; Frank Rexroth, Deutsche Universitätsstiftungen von Prag bis Köln. Die Intentionen des Stifters und die Chancen ihrer Realisierbarkeit im spätmittelalterlichen deutschen Territorialstaat (Beihefte zum Archiv für Kulturgeschichte 34), Köln 1992.

49 Notker Hammerstein, Die Johann Wolfgang Goethe-Universität Frankfurt am Main. Von der Stiftungsuniversität zur staatlichen Hochschule, Bd. I: 1914 bis 1950, Neuwied - Frankfurt a.M. 1989, S. 17.

50 Rainer Christoph Schwinges, Deutsche Universitätsbesucher im 14. und 15. Jahrhundert. Studien zur Sozialgeschichte des Alten Reiches (Veröffentlichungen des Instituts für Europäische Geschichte Mainz, Bd. 123), Stuttgart 1986; Ders., Sozialgeschichtliche Aspekte spätmittelalterlicher Studentenbursen in Deutschland, in: Schulen und Studium im sozialen Wandel des hohen und späten Mittelalters, hg. von Johannes 
Fried (Vorträge und Forschungen, Bd. XXX), Sigmaringen 1986, S. 527-564: Heinz Jürgen Real, Die privaten Stipendienstiftungen der Universität Ingolstadt im ersten Jahrhundert ihres Bestehens. Mit einem Beitrag von Arno Seifert, Das Gregorianum 1494-1600. Frühe Geschichte und Gestalt eines staatlichen Stipendiatenkollegs (Ludovico Maximilianea, Forschungen Bd. 4), Berlin 1972.

51 Vgl. Borgolte (wie Anm. 26) S. 78f. (mit Bezug auf Eberhard F. Bruck), Ders. (wie Anm. 47) bei Anm. 15 (mit Bezug auf K. Schmid, J. Wollasch und O.G. Oexle).

52 Laum (wie Anm. 26) II S. 16f. Nr. 14; dazu: Eberhard F. Bruck, Die Stiftungen für die Toten in Recht, Religion und politischem Denken der Römer, in: Ders., Über römisches Recht im Rahmen der Kulturgeschichte, Berlin - Göttingen - Hamburg 1954, S. 83f., 88 Anm. 16.

$53 \mathrm{Karl}$ Schmid, Stiftungen für das Seelenheil, in: Gedächtnis, das Gemeinschaft stiftet, hg.von Dems. (Schriftenreihe der Katholischen Akademie der Erzdiözese Freiburg), München - Zürich 1985, S. 51-73; vgl.: Ders., Salische Gedenkstiftungen für fideles, servientes und milites, in: Institutionen, Kultur und Gesellschaft im Mittelalter. Festschrift für Josef Fleckenstein, hgg. von Lutz Fenske u.a., Sigmaringen 1984, S. 245264.

54 Michael Borgolte, Gedenkstiftungen in St. Galler Urkunden, in: Memoria (wie Anm. 23) S. 578-602, hier S. 589.

55 Vgl. Oexle, Memoria und Memorialüberlieferung (wie Anm. 23) S. 87ff.: „Gebet als Gabe“.

56 Borgolte (wie Anm. 26) bes. S. 91f., 74.

57 Otto Gerhard Oexle, Die Gegenwart der Toten, in: Death in the Middle Ages, ed. by Herman Braet - Werner Verbeke, Leuven 1983, S. 1977; Ders., Die Gegenwart der Lebenden und der Toten. Gedanken über Memoria, in: Gedächtnis, das Gemeinschaft stiftet (wie Anm. 52) S. 74107.

58 Mauss (wie Anm. 1) S. 176.

59 Hierzu und zum Folgenden: Theodor Straub, Bayern im Zeichen der Teilungen und der Teilherzogtümer (1347-1450), in: Handbuch der bayerischen Geschichte, Bd. II, hgg. von Max Spindler - Andreas Kraus, München 21988, S. 196-287; Ders., Die Ingolstädter Herzogszeit, in: Ingolstadt. Die Herzogsstadt, die Universitätsstadt, die Festung, hrsg. von Theodor Müller - Wilhelm Reissmüller, Bd. I, Ingolstadt 1974, S. 169-219.

60 Wilhelm Störmer, Die innere Konsolidierung der wittelsbachischen Territorialstaaten in Bayern im 15. Jahrhundert, in: Europa 1500. Inte-grationsprozesse im Widerstreit: Staaten, Regionen, Personenverbände, 
Christenheit, hgg. von Ferdinand Seibt - Winfried Eberhard, Stuttgart 1987, S. 175-194.

61 Theodor Straub, Herzog Ludwig der Bärtige von Bayern-Ingolstadt und seine Beziehungen zu Frankreich in der Zeit von 1391 bis 1415 (Münchener Historische Studien. Abteilung Bayerische Geschichte, Bd. 7), Kallmünz 1965; Ders., Die Bayern in Paris zur Zeit der Königin Isabeau de Bavière, in: Festschrift für Max Spindler zu 75. Geburtstag, München 1969, S. 239-282.

62 Straub, Die Ingolstädter Herzogszeit (wie Anm. 58) S. 190-192.

63 Friedrich Wilhelm Fischer, Die Pfarrkirche zur Schönen Unserer Lieben Frau, in: Ingolstadt I (wie Anm. 58) S. 295-355.

64 Ebd., sowie Straub, Die Ingolstädter Herzogszeit (wie Anm. 58) S. 192202.

65 Ebd. S. 192.

66 Zum Folgenden ebd. S. 193-202, sowie: Theodor Straub, Die Hausstiftung der Wittelsbacher in Ingolstadt, in: Sammelblatt des Historischen Vereins Ingolstadt 87 (1978), S. 20-144; Siegfried Hofmann, Die liturgischen Stiftungen Herzog Ludwigs des Gebarteten für die Kirche zur Schönen Unserer Lieben Frau in Ingolstadt. Ein Beitrag zum Verhältnis von Liturgie und Hallenkirche (ebd. S. 145-266). Nach Straub (S. 42f., vgl. Hofmann S. 147) war ,die Begräbnisstiftung von Anfang an Bestandteil der Gesamtplanung (der Kirche) gewesen“.

67 Theodor Müller, Die Wittelsbachische Grablege, in: Ingolstadt I (wie Anm. 58) S. 357-372, Datierung nach Lesung der Inschrift durch Bernhard Bischoff S. 362; dagegen hält Straub, Die Hausstiftung der Wittelsbacher (wie Anm. 65) S. 62, S. 136 Anm. 96, an 1435 fest. - Zur ,Visier" des Künstlers Hans Multscher und zu den anderen Kunstwerken der Grab- und Gedenkstiftung Herzog Ludwigs s.a.: Vavra (wie Anm. 45) S. 127-131.

68 Edition des Stiftungskopialbuches mit 46 umfangreichen Urkunden durch Franz Xaver Buchner, Archivinventare der katholischen Pfarreien in der Diözese Eichstätt, München - Leipzig 1918, Anhang: Die Ingolstädter Schatzurkunden Ludwigs des Gebarteten, S. 655-836. Die für die Stiftung Herzog Ludwigs einschlägigen Schriftstücke sind aber noch nicht vollständig publiziert.

69 Straub, Hausstiftung (wie Anm. 65) S. 44.

70 Buchner (wie Anm. 67) S. 678-684 Nr. II.

71 Ebd. S. 684-688 Nr. III.

72 Ebd. S. 688-696 Nr. IV.

73 Z.B. ebd. S. $679 f$.

74 Ebd. 
75 So Straub, Die Ingolstädter Herzogszeit (wie Anm. 58) S. 193.

76 Straub, Hausstiftung (wie Anm. 65) S. 47-53.

77 Buchner (wie Anm. 67) S. 696-698 Nr. V.

78 Auch die Württemberger haben die Landgeistlichkeit zu regelmäßigen Jahrtagen nach der Stiftskirche ihrer Haupt- und Residenzstadt Stuttgart kommen lassen: Dieter Stievermann, Landesherrschaft und Klosterwesen im spätmittelalterlichen Württemberg, Sigmaringen 1989, S. $145 \mathrm{f}$. Stievermann ordnete den Fürstenjahrtag zutreffend in den Entstehungsproze $ß$ der territorialen Kirchenherrschaft ein, kannte aber offenbar die Ingolstädter Parallele nicht.

79 Auf die ins Fantastische hinübergleitenden Erweiterungen des Stiftungswerkes zwischen 1438 und 1442 gehe ich hier nicht ein.

80 Allerdings haben die Meßstiftungen Ludwigs des Bärtigen und andere liturgische Elemente seines Unternehmens den Herrschaftswechsel überdauert und wurden noch im 16. Jahrhundert beachtet: Straub, Hausstiftung (wie Anm. 65) S. 116.

81 Dazu Borgolte (wie Anm. 47).

82 Vgl. Liermann (wie Anm. 24) S. 124ff., 169ff.

83 Rassem (wie Anm. 25) S. 17.

84 Vgl. Borgolte (wie Anm. 47).

85 Rolf Kießling, Bürgerliche Gesellschaft und Kirche in Augsburg im Spätmittelalter. Ein Beitrag zur Strukturanalyse einer oberdeutschen Reichsstadt, Augsburg 1971, S. 245-287.

86 Marlene Besold-Backmund, Stiftungen und Stiftungswirklichkeit. Studien zur Sozialgeschichte der beiden oberfränkischen Kleinstädte Forchheim und Weismain (Schriften des Zentralinstituts für fränkische Landeskunde und allgemeine Regionalforschung an der Universität Erlangen-Nünberg, Bd. 27), Neustadt an der Aisch 1986.

87 Vgl. Borgolte (wie Anm. 26) S. 78f. (unter Bezug auf die Deutungsversuche von Bruck).

88 Vgl. Borgolte (wie Anm. 47) bei Anm. 25-32 (unter Bezug auf die Schule von U. Stutz).

89 Die Zunahme caritativer Stiftungen seit dem 12./13. Jahrhundert wird mit der Verbürgerlichung des Stiftungswesens erklärt: Harry Kühnel, Sinn und Motivation mittelalterlicher Stiftungen, in: Materielle Kultur und religiöse Stiftung (wie Anm. 42) S. 5-12, S. 5 (nach B. Geremek).

90 Zum Buch Liermanns s.o. Anm. 24.

91 Harry Ebersbach, Handbuch des Stiftungsrechts, Göttingen 1972, S. 4

92 Dies war schon 1972 festzustellen, als die stiftungsrechtlichen Vorschriften der $\S 80-88$ BGB noch galten: Ebd. S. 327.

93 Zivilgesetzbuch der Deutschen Demokratischen Republik sowie an- 
grenzende Gesetze und Bestimmungen. Textausgabe mit Anmerkungen und Sachregister, hg. vom Ministerium der Justiz, Berlin 1978. Im Einführungsgesetz zum Zivilgesetzbuch der Deutschen Demokratischen Republik vom 19. Juni 1975 wurde $§ 9$ den Stiftungen gewidmet (ebd. S. 114); allerdings regeln die Bestimmungen lediglich die Rechtstellung vorhandener Stiftungen und sehen keine Neuerrichtungen vor.

94 Hardtwig (wie Anm. 15). 


\section{Michael Borgolte}

1948 in Braunschweig geboren.

Studium der Geschichte, Germanistik und Philosophie.

Promotion 1975 in Münster/Westfalen.

Wissenschaftlicher Assistent an der Universität Freiburg/Breisgau.

1981 Habilitation in Freiburg/Breisgau.

Lehrtätigkeit als Dozent und Professor an den Universitäten

Freiburg, Basel, Frankfurt/M. und Bamberg.

Seit Dezember 1991 Professor für Mittelalterliche Geschichte an der Humboldt-Universität zu Berlin.

Wichtigste Veröffentlichungen

Der Gesandtenaustausch der Karolinger mit den Abbasiden und mit den Patriarchen von Jerusalem, München 1976

Geschichte der Grafschaften Alemanniens in fränkischer Zeit, Sigmaringen 1984

Die Grafen Alemanniens in merowingischer und karolingischer Zeit. Eine Prosopographie, Sigmaringen 1986

Subsidia Sangallensia, St. Gallen 1986 (Hrsg.)

Litterae Medii Aevi, Sigmaringen 1988 (Hrsg.)

Petrusnachfolge und Kaiserimitation. Die Grablegen der Päpste, ihre Genese und Traditionsbildung, Göttingen 1989

Die mittelalterliche Kirche, München 1992

Mittelalterforschung nach der Wende, im Druck (Hrsg.) 


\section{In der Reihe Öffentliche Vorlesungen sind erschienen:}

1 Volker Gerhardt

Zur philosophischen Tradition der HumboldtUniversität

2 Hasso Hofmann

Die versprochene Menschenwürde

$3 \quad$ Heinrich August Winkler

Von Hitler zu Weimar

Die Arbeiterbewegung und das Scheitern der ersten deutschen

Demokratie

Es erscheinen demnächst:

$5 \quad$ Wilfried Nippel

Max Weber und die Althistorie seiner Zeit

$6 \quad$ Heinz Schilling

Am Anfang waren Luther, Loyola und Calvin ein religionssoziologisch-entwicklungsgeschichtlicher Vergleich

$7 \quad$ Hartmut Harnisch

Adel und Großgrundbesitz im ostelbischen Preußen 1800 - 1914 